\title{
Design of the Traction Battery for a Formula SAE Racing Car
}

\author{
Federico Baronti*, Daniele Calderini ${ }^{\S}$, Gianluca Caposciutti ${ }^{\S}$, Andrea Gassani ${ }^{\S}$ Riccardo Moras $^{\S}$ and Roberto Saletti* \\ *Dipartimento di Ingegneria dell’Informazione, Università di Pisa, I-56122 Pisa, Italy E-mail: f.baronti@iet.unipi.it

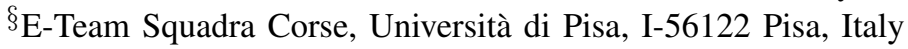

\begin{abstract}
This paper describes the design of the traction battery for the new electric Formula SAE vehicle of the University of Pisa. A model based design methodology extended to the mechanical, electrical and thermal domains was applied to find the best trade-off between the battery weight and the maximum power available at the wheel. The designed battery configuration was validated by means of electrical and thermal simulations.
\end{abstract}

\section{INTRODUCTION}

Formula SAE (FSAE) is an international racing competition created by the Society of Automotive Engineers in 1978. It is held every year in several locations around the world and challenges university students. They are asked to conceive, design and drive a small, formula style vehicle complying with the official FSAE rules [1]. With the growing interest for sustainable transportation, this competition, originally reserved for combustion engine vehicles, was extended to electric ones in 2010, in order to foster education and research on electric mobility [2]-[4]. Each round of the competition consists of static and dynamic events. During the former, students present and discuss their design choices with experts from academy and industry. Both technical and economical aspects are evaluated with the view to a small series production of the designed vehicle. Dynamic events include an acceleration test and an endurance race of approximately $20 \mathrm{~km}$.

The onboard energy storage system (ESS) dramatically affects the dynamic performance of an electric FSAE car, as the ESS weight is a significant portion of the overall vehicle weight. Thus, its optimization under the constraint that the ESS provides the high power requested during the acceleration test and stores enough energy to complete the endurance race, is a fundamental design goal for an electric FSAE car. This goal can be achieved by using the Lithium Polymer (LiPo) battery technology, which provides a very good trade-off between power and energy densities, compared to other ESS technologies [5]. However, this battery technology is famous for its fragility, as it cannot withstand operation outside well defined voltage and temperature ranges. The traction battery of a FSAE electric car has to be as light as possible and provide suitable acceleration capabilities. Thus, it is subjected to high C-rate discharging currents, which are likely to create thermal problems if the mechanical assembly of the battery cells, as well as the cooling system, are not properly designed and validated.

The objective of this paper is to describe the methodology used to size, design and validate the traction battery of the FSAE racing car, which is being developed at the University of Pisa (Italy). An important point of this work is the adoption of a model-based design approach that integrates all the engineering fields involved in the application, i.e., mechanical, electrical and thermal fields. In fact, the optimum sizing of the battery was obtained by means of a dynamic model of the FSAE car running an endurance race. The correct behavior of the battery was then verified by electrical simulations, which exploit an accurate model of LiPo cells. Finally, a thermal model of the battery cells considering the designed mechanical layout and the cooling system was developed and Computational Fluid Dynamics (CFD) analyses were performed to verify that the cell temperatures remain in the safe range during the endurance race.

\section{BATTERY DESIGN}

The exploration of the design space of the ESS is bounded by the relevant FSAE Rules [1]. Their purpose is to guarantee the safety of the students during the car assembly and the dynamic events. In the following, we report the most important constraints that apply to the design of the ESS for a FSAE racing car [1].

- All types of accumulators except molten salt and thermal batteries are allowed. E.g.: Batteries, Supercapacitors, etc. Fuel cells are prohibited.

- The maximum permitted voltage is $600 \mathrm{~V}$.

- $\quad$ Each battery segment must contain a maximum energy of $12 \mathrm{MJ}$ and its static voltage must be less than $120 \mathrm{~V}$.

- $\quad$ The maximum power drawn from the battery must not exceed $85 \mathrm{~kW}$.

- A Battery Management System (BMS) is mandatory to continuously measure the voltage of every cell and the temperature of at least $30 \%$ of the cells.

\section{A. Battery Sizing}

A state of the art analysis of other FSAE electric cars was carried out as a starting point for the exploration of the ESS design space. This analysis pointed out that the LiPo battery technology is the common choice of the FSAE top teams for the implementation of the onboard ESS. Compared to other ESS technologies, LiPo battery cells provide indeed a very good trade-off between power and energy densities, at the expense of a higher sensitivity to overcharge, deep discharge and overtemperature [5]. As the weight of the ESS has a significant impact on the performance of the vehicle, 


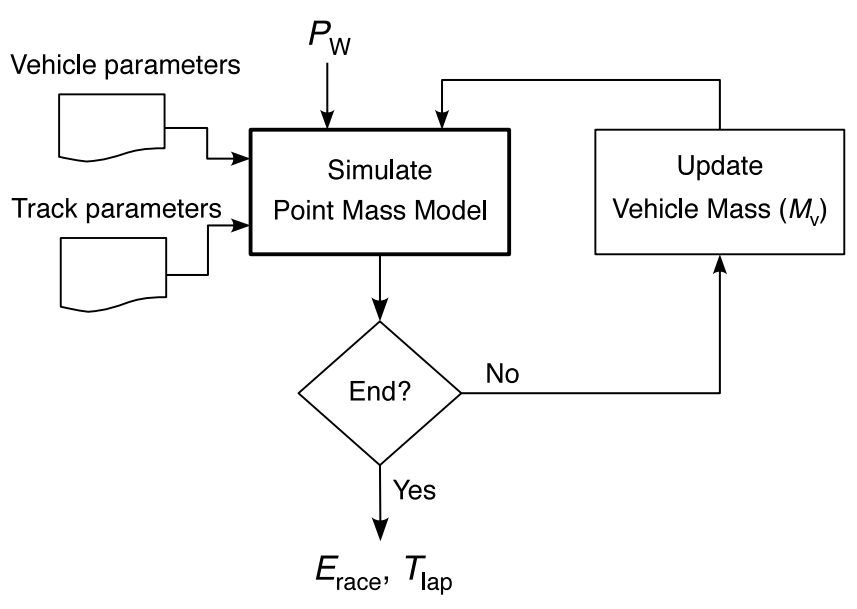

Fig. 1. Flow diagram of the battery size optmization.

we selected this battery technology, though it poses severe challenges on its electrical and thermal management that have to be properly faced.

The preliminary step for designing the ESS was to determine the battery energy size $E_{\mathrm{B}}$ needed to complete the race, which minimizes the lap time. On the one hand, decreasing $E_{\mathrm{B}}$ and consequently the vehicle mass $M_{\mathrm{V}}$ (which includes the ESS mass) makes the car faster when cornering. On the other hand, it requires a reduction of the maximum power at the wheels $P_{\mathrm{W}}$ (to complete the race), which makes the car slower when going straight. Thus, we expect the existence of an optimum value of $E_{\mathrm{B}}$. To find this value, a simple but accurate simulation environment was developed using the Mathematica ${ }^{\circledR}$ software. It is based on a simple point mass model of the vehicle, which is assumed to follow an assigned path, driven by an ideal driver. The ideal driver imposes the optimal speed profile, computed considering $M_{\mathrm{V}}, P_{\mathrm{W}}$, the aerodynamics forces and the tires characteristics. The power at the battery terminals is obtained considering the inverter efficiency and the electric motor efficiency map. The model was used to simulate the endurance race on the circuit of the Formula Student Germany, whose track layout and parameters have been extracted from the data logged during the FSAE event in 2011 [6]. The outputs of the simulation are the energy used to complete the race $E_{\text {race }}$ and the time needed to travel one lap of the track $T_{\text {lap }}$, as well as the power at the battery terminals.

The optimization procedure consisted in varying $P_{\mathrm{W}}$ from $26 \mathrm{~kW}$ to $40 \mathrm{~kW}$ and in finding for each $P_{\mathrm{W}}$ value, the corresponding value of $E_{\mathrm{B}}$ that guarantees to complete the race with a desired safety margin. As $E_{\text {race }}$ depends on the vehicle mass, which in turn depends on $E_{\mathrm{B}}$, the iterative algorithm described in (1) was used to obtain $E_{\mathrm{B}}$ for each analyzed value of $P_{\mathrm{W}}$, as shown in the flow diagram of Fig. 1. The "Simulate Point Mass Model" block receives $P_{\mathrm{W}}$ and $M_{\mathrm{V}}$ as input and the vehicle and track data as parameters. $P_{\mathrm{W}}$ is set at the beginning of the procedure, whereas $M_{\mathrm{V}}$ is updated at each iteration by the "Update Vehicle Mass". The procedure ends when the absolute difference between two consecutive values of $E_{\text {race }}$ is less than $100 \mathrm{Wh}$. The vehicle mass is the sum of a constant term $M_{\mathrm{C}}$, which includes all the onboard masses except the ESS, and the ESS itself. The experience of

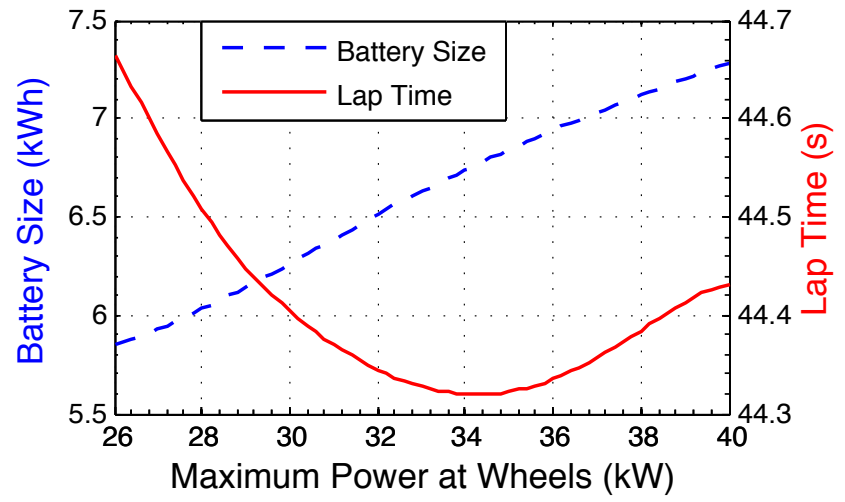

Fig. 2. Battery size and lap time as function of the maximum power at the wheels.

the previous FSAE cars leads to an estimate of $M_{\mathrm{C}}=200 \mathrm{~kg}$ [6]. The ESS mass is computed by dividing $E_{\mathrm{B}}$ by the ESS energy density $\rho_{\mathrm{B}}$. We assumed $\rho_{\mathrm{B}}=100 \mathrm{~W} \mathrm{~h} \mathrm{~kg}^{-1}$, which is a reasonable value for the LiPo battery technology considering the overhead due to the assembling of the battery pack. The next value of $E_{\mathrm{B}}$ is obtained as the simulation result $E_{\text {race }}$ times a factor $k$, with $k>1$, which determines the amount of residual energy stored in the battery at the end of the race. By setting $k=1.1$, we avoid the deep discharge of the battery as its state of charge at the end of the endurance event is still $10 \%$.

$$
\left\{\begin{array}{l}
E_{\mathrm{B}}(0)=7 \mathrm{kWh} \\
E_{\mathrm{B}}(i)=k E_{\mathrm{race}}(i-1) \\
M_{\mathrm{V}}=M_{\mathrm{C}}+E_{\mathrm{B}}(i) / \rho_{\mathrm{B}}
\end{array}\right.
$$

Figure 2 shows the battery size $E_{\mathrm{B}}$ and the lap time $T_{\text {lap }}$, as a function of $P_{\mathrm{W}}$. The lap time is minimized with $P_{\mathrm{W}}=34.4 \mathrm{~kW}$, which yields $T_{\text {lap }}=44.33 \mathrm{~s}$ and $E_{\mathrm{B}}=$ $6.8 \mathrm{kWh}$. This battery size is in good agreement with those found in the survey of other electric FSAE cars, which lie in the range $5 \mathrm{~kW}$ h to $7 \mathrm{~kW} \mathrm{~h}$. Figure 2 also shows that decreasing $P_{\mathrm{W}}$ down to $26 \mathrm{~kW}$, the lap time increases of less than $1 \%$ and the required stored energy $E_{\mathrm{B}}$ is reduced to $5.8 \mathrm{kWh}$. This introduces a valuable flexibility, which can be exploited in completing the design of the battery. To this end, given the number of series-connected cells $N$, the nominal voltage $V_{\mathrm{n}}=3.7 \mathrm{~V}$ of a LiPo cell, the cell capacity $C_{\mathrm{n}}$, we express $E_{\mathrm{B}}$ as in (2). It is useful to relate $E_{\mathrm{B}}$ to the maximum voltage at the battery's terminals $V_{\max }=4.2 N$, being $4.2 \mathrm{~V}$ the voltage of a fully charged LiPo cell. As $V_{\max }$ is limited by the FSAE rules to $600 \mathrm{~V}$, the maximum number of LiPo cells that can be serially connected is 142. In this configuration, the cell capacity to store the optimum amount of energy $7 \mathrm{kWh}$ is $12.9 \mathrm{Ah}$.

$$
E_{\mathrm{B}}=N V_{\mathrm{n}} C_{\mathrm{n}}=\frac{3.7}{4.2} V_{\max } C_{\mathrm{n}}
$$

For the selection of the battery cell, we also need to estimate the maximum discharging continuous and peak powers of the battery. The maximum discharging power is required during the acceleration event, in which the maximum allowed (by FSAE rules) power $P_{\mathrm{B} \text {,peak }}=85 \mathrm{~kW}$ is requested from the battery for a short time (less than $10 \mathrm{~s}$ ). As $P_{\mathrm{W}}$ was limited in our design exploration to $40 \mathrm{~kW}$, we end up to a 


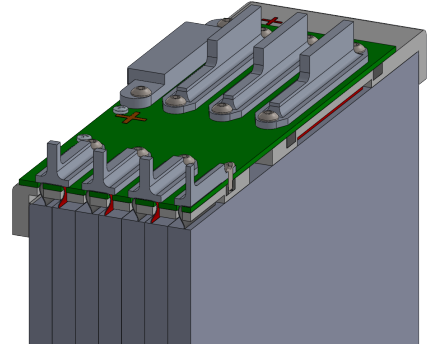

(a)

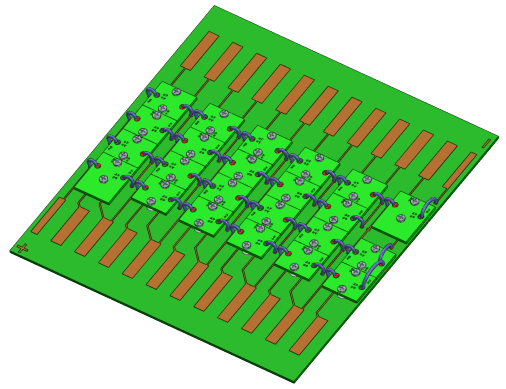

(b)

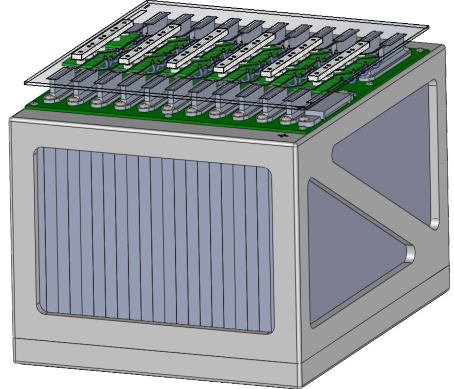

(c)

Fig. 3. (a) Cells series-connection detail. (b) Segment configuration. (c) BMS slave boards connection.

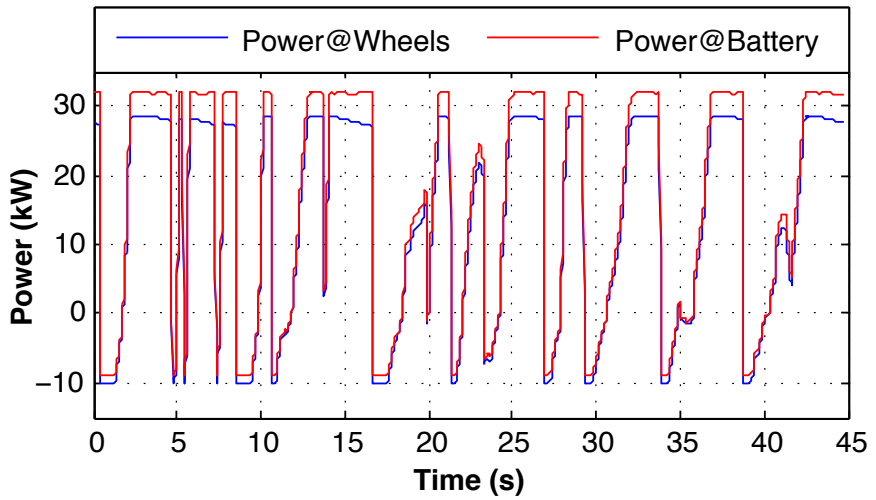

Fig. 4. Power at wheels and at the battery's terminals during one lap.

maximum "continuous" discharging power $P_{\mathrm{B}, \text { cont }} \simeq 45 \mathrm{~kW}$, taking into account all the energy losses from the battery to wheels (i.e., approximately an $88 \%$ efficiency from the battery to the wheels). From $E_{\mathrm{B}}$ and the continuous and peak values of the maximum discharge power, it is possible to estimate the continuous and peak values of the maximum discharge current in terms of C-rate, Crate $_{\text {cont }}$ and Crate $_{\text {peak }}$, respectively.

$$
\begin{aligned}
& P_{\mathrm{B}, \text { cont }}=N V_{\mathrm{n}} \frac{C_{\mathrm{n}}}{1 \mathrm{~h}} \text { Crate }_{\mathrm{cont}}=\frac{E_{\mathrm{B}}}{1 \mathrm{~h}} \text { Crate }_{\mathrm{cont}} \\
& P_{\mathrm{B}, \text { peak }}=N V_{\mathrm{n}} \frac{C_{\mathrm{n}}}{1 \mathrm{~h}} \text { Crate }_{\text {peak }}=\frac{E_{\mathrm{B}}}{1 \mathrm{~h}} \text { Crate }_{\text {peak }}
\end{aligned}
$$

From (3), we obtain Crate $_{\text {cont }} \approx 7$ and Crate $_{\text {peak }} \approx 13$, values that must be sustained by the cell to be selected. This means that high power cells are needed, thus, confirming the initial choice of the LiPo battery technology. A very good trade-off between power and energy densities is indeed found in the $12 \mathrm{~A} \mathrm{~h} \mathrm{LiPo} \mathrm{cell} \mathrm{(SLPB70205130P)} \mathrm{from} \mathrm{Kokam}$ Ultra-High-Power Series. The maximum continuous and peak discharge (for less than $20 \mathrm{~s}$ ) currents are 15 and $20 \mathrm{C}$ rate, respectively, and the mass is $350 \mathrm{~g}$, thus satisfying the previously stated requirements. The cell case is the pouch type.

\section{B. Battery assembly}

To complete the design of the traction battery, we have to decide how to assemble the selected elemental cells to store the required energy with the constraints imposed by the FSAE rules. We chose to partition the battery into 6 segments of 23 cells each. The overall number of series-connected $12 \mathrm{~A} \mathrm{~h}$ cells is 138, which leads to a maximum voltage of $579.6 \mathrm{~V}$ and a stored energy of $6127.2 \mathrm{Wh}$. According to Fig. 2, the maximum power at wheels is thus limited to $29 \mathrm{~kW}$. This leads to $T_{\text {lap }}=44.47 \mathrm{~s}$, which is very close to the minimum value. Each segment has a maximum voltage of $96.6 \mathrm{~V}$ and stores approximately $3.7 \mathrm{MJ}$, thus complying with the FSAE rules.

Figure 3 shows the designed assembly of a segment. In particular, Fig. 3(a) shows the connection between adjacent cells, which is obtained by folding the tabs of two adjacent cells and assuring the electrical connection by pressing them against an insulating substrate by means of an aluminum tab. The shape of the latter is designed so that it can be contacted from a an overlaid board (see Fig. 3(b)), which hosts the slave boards of the BMS. The adopted BMS has indeed a hierarchical architecture, in which the voltage and temperature of each cell are measured by a dedicated slave board. The slave board are chained and eventually connected to the master BMS unit. This assembly provides a reliable electrical connection between the serially connected cells and a simple and effective connection to the BMS. The goal is a reduction of the production costs in a serial production of the vehicle, which is positively evaluated by the FSAE judges.

The overall mass of the designed battery is estimated to be around $65 \mathrm{~kg}$. This is in good agreement with the initial hypothesis on the energy density $\rho_{\mathrm{B}}=100 \mathrm{Wh} \mathrm{kg}^{-1}$ and $E_{\mathrm{B}}=6.1 \mathrm{~kW}$. Finally, Fig. 4 shows the power at the battery's terminals (for $P_{\mathrm{W}}=29 \mathrm{~kW}$ ) during one lap of the race, which is used for the electrical and thermal simulations described in the following Sections.

\section{ElECTRICAL SimUlations}

The process described above allowed us to design the battery configuration that satisfies the requirements of the target application in terms of power and energy. The main issue is now to verify that, during the endurance event, the battery remains in the safe operating area, in terms of the voltage and temperature of the cells, while providing the requested power. This issue has been addressed by electrical and thermal simulations. The former exploits an accurate model of LiPo cells developed in [7], which is capable of reproducing the dynamic behavior of the cell voltage faithfully, given the cell current. Thermal simulations are based on a CFD model of 


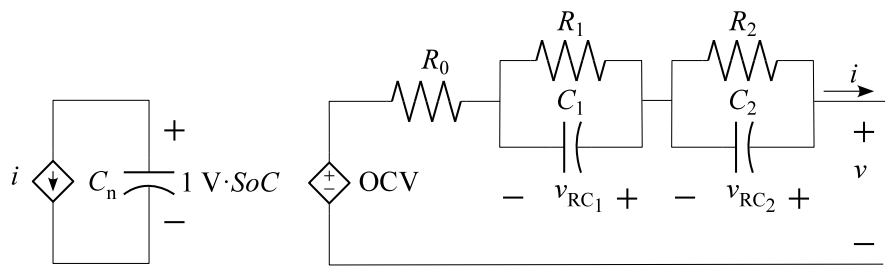

Fig. 5. Electrical model of a LiPo cell.

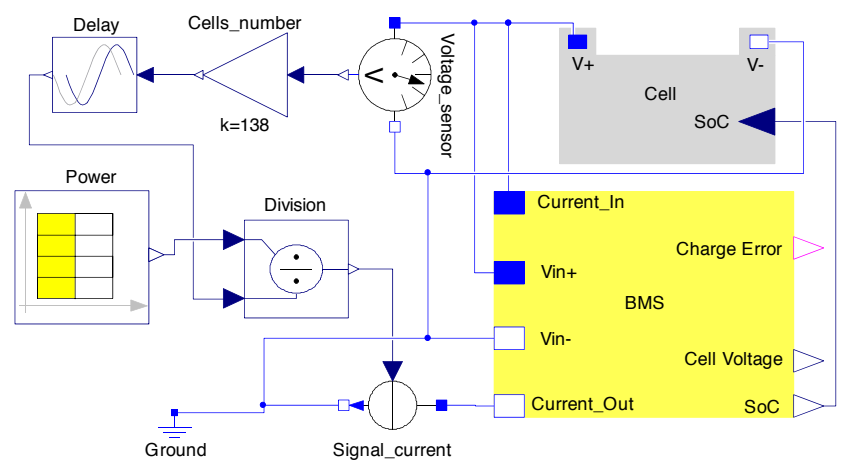

Fig. 6. Block diagram of the model used for the electrical simulations in Dymola.

the battery, which takes into account the battery assembly geometry, as well as the cooling system. The heat generated in the battery is due to the power dissipated by the cells, as obtained by the electrical simulations.

\section{A. Electrical Model}

Figure 5 shows an equivalent electrical model of a cell widely accepted in the literature [8]. The left hand side of the model in Fig. 5 reproduces the State-of-Charge (SoC) of the cell by means of Coulomb-counting of the cell current $i$. The right hand side reproduces the cell terminal voltage $v$, as a sum of the Open Circuit Voltage (OCV), which is a non linear function of the SoC, and two relaxation voltages $v_{\mathrm{RC} 1}$ and $v_{\mathrm{RC} 2}$. The corresponding time constants are in the order of tens and hundreds of seconds, respectively [9]. The model parameters are not constant as they depend on the SoC and the temperature of the cell and are managed by a 2-dimensional Look-Up-Table (LUT). The relationship between OCV and SoC is also recorded in a LUT.

The electrical model shown in Fig. 5 was fully characterized for a $1.5 \mathrm{Ah}$ cell belonging to same family of the cells used in the battery described in this work [7]. We note that a $12 \mathrm{Ah}$ cell can be modeled by the parallel of 8 cells of the same chemistry with a capacity of $1.5 \mathrm{Ah}$. Thus, the model parameters related to the $12 \mathrm{Ah}$ were obtained by scaling a factor 8 those of the $1.5 \mathrm{Ah}$, i.e., dividing the resistive terms and multiplying the capacitive ones. The SoC-OCV relationship depends only on the battery chemistry and is invariant with the cell capacity.

\section{B. Electrical Simulations}

The electrical model was implemented in the Dymola simulator, a multi-domain simulation software. Only one cell of the battery is simulated as all the cells are identical and

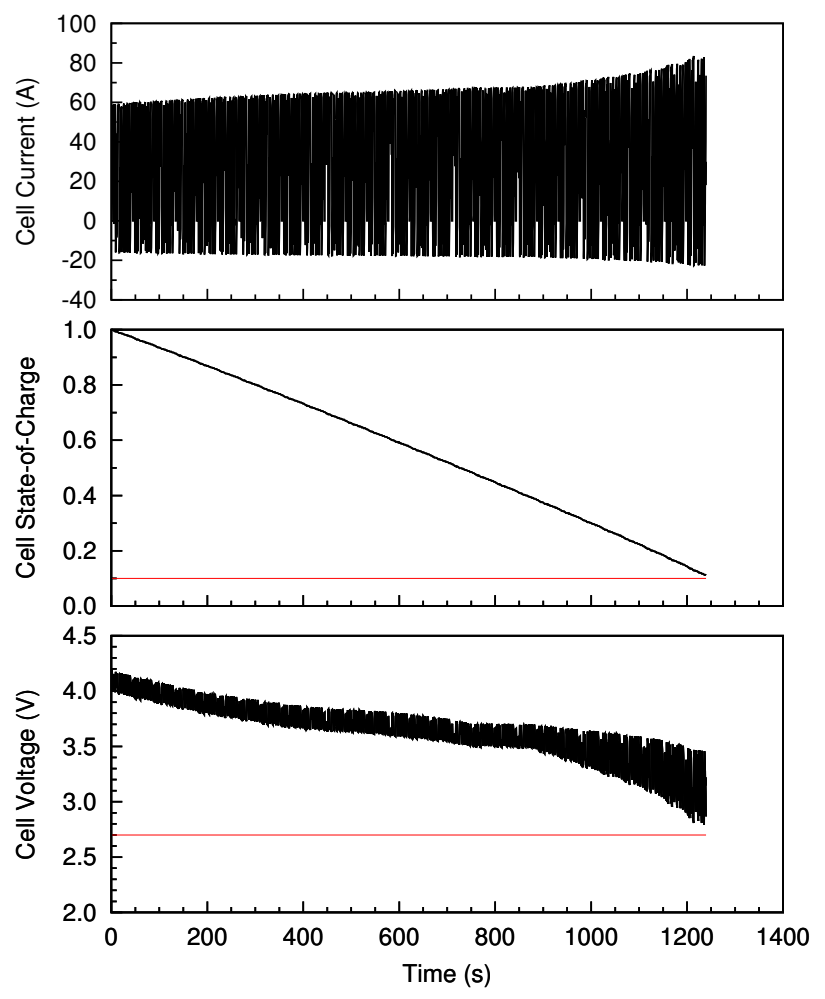

Fig. 7. Cell current, SoC and voltage during the endurance race supposing a maximum power of $29 \mathrm{~kW}$ available at the wheels. The red lines show the safety limits.

are subjected to the same current. Thus, the voltage of the battery is 138 time that of a single cell. The block diagram of the simulation set-up is shown in Fig. 6. It includes the "Cell" block that accounts for the right hand side of the electrical model shown in Fig. 5. Its left hand side is instead incorporated in the "BMS" block, which also controls that the voltage and current of the cell remain in their proper safe ranges. The input of the simulation is the battery power profile shown in Fig. 4, which is repeated for all the 28 laps of the endurance race. The battery current is computed at each simulation step by dividing the current input power by the battery voltage computed at the previous simulation step.

The behavior of the cell voltage during the endurance race is shown in the bottom chart of Fig. 7. It is worth noting that the cell voltage always remains above the discharge cut-off voltage (red line), which is specified by the producer as $2.7 \mathrm{~V}$. Also, the battery SoC at the end of the endurance race is around $10 \%$, as expected from the analysis carried out in Section II-A to size the battery. The top chart of Fig. 7 shows the battery current that increases to maintain the same power profile lap after lap when the battery voltage decreases. From the battery current, we calculated the power losses in each cell as the power dissipated by the resistor $R_{0}$ in the electrical model of Fig. 5. For this computation, we considered $R_{0}$ constant and equal to $2.2 \mathrm{~m} \Omega$. These losses are the bridge to the next thermal domain analysis.

\section{Thermal Simulations}

The objective of this section is to verify that each cell of the battery remains below $55^{\circ} \mathrm{C}$, a safe temperature for the used battery technology, during all the endurance race. To this 


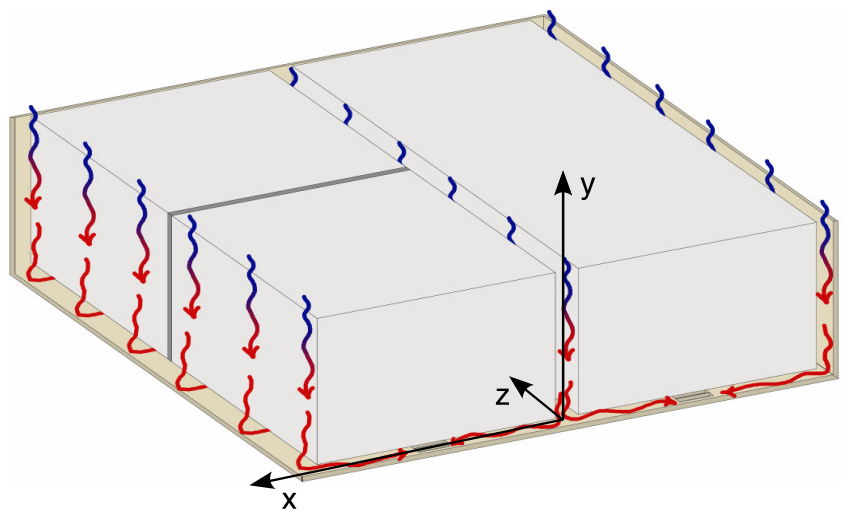

Fig. 8. Simplified 3D view of the battery assembly. The cell analyzed is that in dark grey.

end, we developed a thermal model of the whole battery, which accounts for the physical assembly of the six battery segments (see Fig. 3(c)) and the cooling system. The six segments are arranged in two rows ( $3 \times 23$ cells per row) inside a carbonfiber-reinforced polymer case, which is located under the pilot seat. Fig. 8 shows the battery layout, together with the cooling mechanism. The latter is based on four cooling air channels: one in the middle of the case, two on the outer sides and one below the cells. The air inlet is located in the upper part of the case above the middle and lateral channels and the outlet (a $30 \times 7 \mathrm{~mm}^{2}$ hole) is placed under every cell. In this way, the air flux is in countercurrent with natural convection to promote flow turbulence. The air flux is distributed by small fans, which are sized to have approximately $5 \times 10^{-4} \mathrm{~kg} \mathrm{~s}^{-1}$ cooling air for every cell.

The key point in developing the thermal model is finding the right trade-off between accuracy in predicting the maximum cell temperature over the endurance race and computational complexity. Two considerations can be derived from the physical layout of the battery. First, the most critical cells are those located in the middle of the case. Second, the system is symmetric about the yz plane, which lies in the center of the middle air channel, whose width is twice that of the side channels (see Fig. 9). Thus, we can simplify the problem by considering only the cell located in the center of a row. Moreover, we assume that heat exchange occurs only between the lateral and bottom walls of the cell and the corresponding air channels, while all the other surfaces are considered adiabatic, as shown in Fig. 9. Thermal generation within the cell is assumed to be uniformly distributed in space and is computed as the power losses in a cell divided by its volume, i.e., $210 \times 132 \times 7.5 \mathrm{~mm}^{3}$ (width $\mathrm{x}$ height $\mathrm{x}$ thickness). The thermal parameters of the cell are reported in Table I [10]. Finally, we note that all the assumptions made in developing the thermal model lead to overestimate the maximum cell temperature. Thus, if the simulated maximum cell temperature is below the safe one, we can expect that all the battery cells remain safely below the maximum permitted temperature.

Thermal simulations were carried out with the ANSYS FLUENT v14.0 software. The cell temperature behavior was simulated for a complete endurance race, which lasts around $1245 \mathrm{~s}$. The initial condition is the cell in the equilibrium with

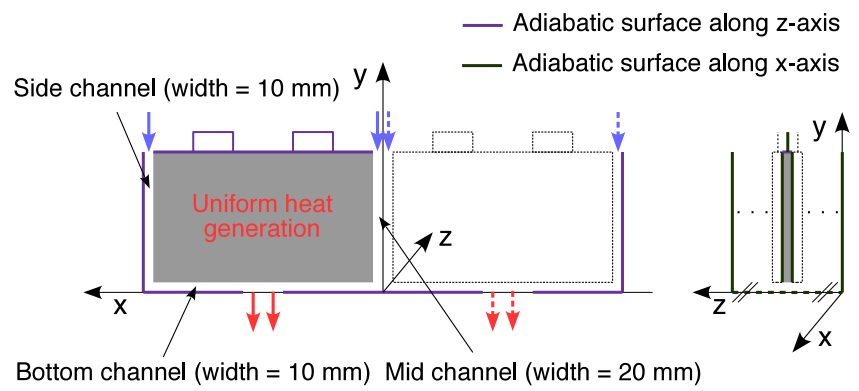

Fig. 9. Schematic representation of the used thermal model.

TABLE I. THERMAL PARAMETERS OF A LITHIUM-ION CELL

\begin{tabular}{lccc}
\hline Parameter & Unit & Typical range & Used value \\
\hline Thermal conductivity & $\mathrm{W} /(\mathrm{m} \mathrm{K})$ & $0.40-0.85$ & 0.66 \\
Specific heat at constant pressure & $\mathrm{J} /(\mathrm{kg} \mathrm{K})$ & $650-950$ & 800 \\
Density & $\mathrm{kg} / \mathrm{m}^{3}$ & $1700-2500$ & 2100 \\
\hline
\end{tabular}

the air at $35^{\circ} \mathrm{C}$, a reasonable value near the asphalt in summer. The temperature map at the end of the endurance race in the middle section (parallel to the xy plane) of the simulated cell is shown in Fig. 10. As expected, the highest temperature is in the center-top of the cell, but safely below the maximum value of $55^{\circ} \mathrm{C}$.

With a convergence analysis based on successive mesh refinements, we estimated a maximum temperature error of about $1^{\circ} \mathrm{C}$. To validate the CFD solution, we computed the analytical solution of the thermal problem in the middle section (parallel to the xy plane) of the cell. To this end, we used the cell thermal parameters reported in Table I and we modeled the heat transfer at the cell boundaries with constant coefficients calculated for rectangular plates [11]. The thermal generation function was approximated by its average value, plus a sinusoidal component extracted from its Fourier series. This approximation made it possible to apply the Green's technique [12]. The analytical solution computed within the cell is compared to the CFD one in Fig. 11. The figure shows a good agreement between the two solutions, in particular in the

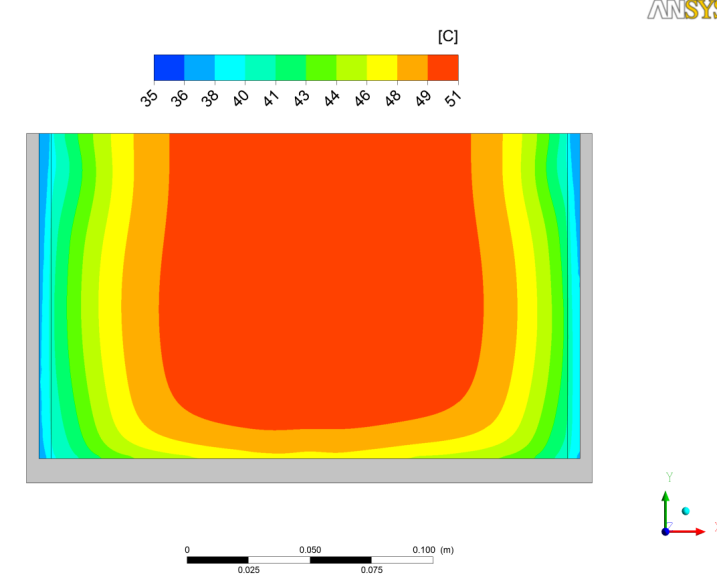

Fig. 10. Temperature map at the end of the endurance race in the middle section (parallel to the xy plane) of the simulated cell. 


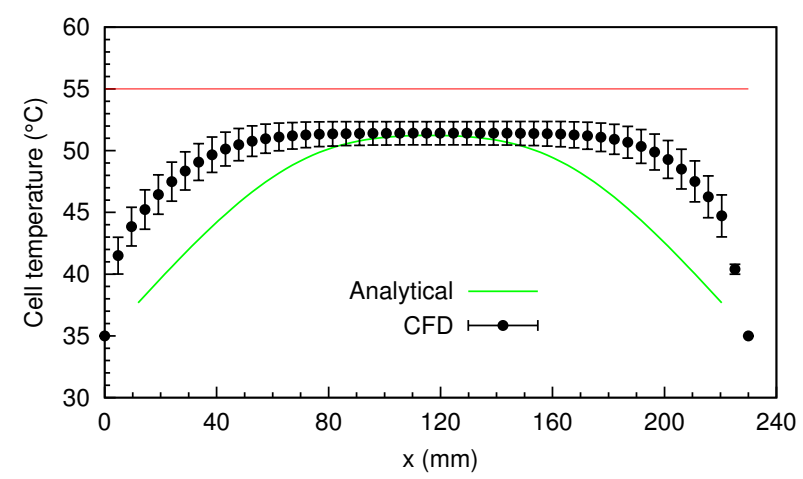

Fig. 11. Cell temperature variation along the $\mathrm{x}$-axis at the end of the endurance race.

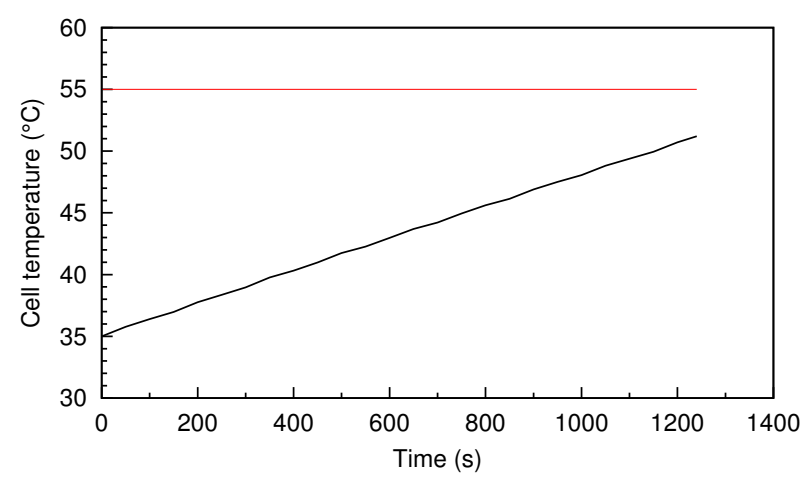

Fig. 12. Temperature in the center of cell during the endurance race.

center of the cell, where the approximations made to calculate the analytical solution on the boundary heat transfer are less relevant. Both solutions agree that the maximum temperature present in the center of the cell is about $51^{\circ} \mathrm{C}$, a value safely below the maximum permitted.

Finally, Fig. 12 shows the cell temperature behavior during the endurance race. It confirms that the cell temperature at the end of the endurance race does not exceed the maximum permitted value, also including computational errors, starting from an initial temperature of $35^{\circ} \mathrm{C}$. In fact, the net result of the thermal simulations is that the cell temperature raises of $16{ }^{\circ} \mathrm{C}$ during the race. We note that the final value of the cell temperature can be adjusted by controlling the initial temperature of the battery, a technique permitted by the FSAE rules.

\section{CONClusion}

This paper has described the design of a Lithium-ion battery to be used as energy storage for the electrical propulsion of a FSAE electric race car. The main specifications of the battery have been determined starting from the FSAE rules. Then, LiPo high-power cells have properly been chosen according to the power and energy needed by the application. These requirements have been obtained with an optimization procedure based on a dynamic model of the vehicle. The two fundamental issues related to the electrical and thermal behavior of the battery have been addressed with electrical and thermal simulations that take into account the power load profile of the battery calculated with the dynamic model of the vehicle. The simulations demonstrate that the endurance race can be completed without safety risks and even without impairing the health of the battery, which remains above $10 \%$ of SoC and do not enter the deep discharge region, if the maximum mechanical power is limited to $29 \mathrm{~kW}$. In this case the cooling system maintains the most critical cell maximum temperature below the limit of $55^{\circ} \mathrm{C}$.

The final conclusion is that the design of a battery system for a demanding application such as the FSAE electric race cars can efficiently be solved with an effective integration of multi-disciplines competences and multi-dimensional modeling that spans over the mechanical, electrical and thermal aspects of the system. Up-to-date simulation tools are also needed to validate the design choices before prototyping the battery system.

\section{ACKNOWLEDGMENT}

The authors would like to acknowledge Dr. Francesco Bucchi, Prof. Massimo Ceraolo, Dr. Gabriele Fantechi, and Prof. Massimo Guiggiani for their valuable contribution to this work.

\section{REFERENCES}

[1] 2014 Formula SAE Rules. [Online]. Available: http://students.sae.org/ cds/formulaseries/rules/

[2] F. J. Sanchez-Alejo, F. Aparicio, M. A. Alvarez, and E. Galindo, "The developing of personal and professional skills in automotive engineers through university competitions," in IEEE EDUCON 2010 Conf. IEEE, 2010, pp. 1491-1498.

[3] I. Chang, N. Kim, D. Lee, Cha, and S. Won, "Designing and manufacturing of Formula SAE-Hybrid racecar for a new engineering education program," in 2010 IEEE Veh. Power Propuls. Conf. IEEE, Sep. 2010, pp. 1-6.

[4] E. Carraro, M. Degano, M. Morandin, and N. Bianchi, "Formula SAE electric competition: Electrical motor design," in 2013 Int. Electr. Mach. Drives Conf. IEEE, May 2013, pp. 1142-1148.

[5] M. S. Whittingham, "History, Evolution, and Future Status of Energy Storage," Proc. IEEE, vol. 100, no. Special Centennial Issue, pp. 1518-1534, May 2012.

[6] F. Baronti, A. Lazzeri, R. Roncella, R. Saletti, and S. Saponara, "Design and Characterization of a Robotized Gearbox System Based on Voice Coil Actuators for a Formula SAE Race Car," IEEE/ASME Trans. Mechatronics, vol. 18, no. 1, pp. 53-61, Feb. 2013.

[7] F. Baronti, G. Fantechi, E. Leonardi, R. Roncella, and R. Saletti, "Enhanced model for Lithium-Polymer cells including temperature effects," in IECON 2010 - 36th Annu. Conf. IEEE Ind. Electron. Soc. IEEE, Nov. 2010, pp. 2329-2333.

[8] M. Chen and G. Rincon-Mora, "Accurate Electrical Battery Model Capable of Predicting Runtime and IV Performance," IEEE Trans. Energy Convers., vol. 21, no. 2, pp. 504-511, Jun. 2006.

[9] F. Baronti, W. Zamboni, N. Femia, H. Rahimi-Eichi, R. Roncella, S. Rosi, R. Saletti, and M.-Y. Chow, "Parameter identification of Li-Po batteries in electric vehicles: A comparative study," in 2013 IEEE Int. Symp. Ind. Electron. IEEE, May 2013, pp. 1-7.

[10] M. Muratori, "Thermal characterization of Lithium-Ion battery cell," PhD Thesis, University of Pisa, 2008.

[11] Y. A. Cengel, Introduction to Thermodynamics and Heat Transfer. McGraw-Hill, Science, 1997.

[12] M. N. Ozisik, Heat conduction, 2nd ed. John Wiley and Sons Inc., 1993. 